\title{
Propagation-induced transition from slow to fast light in highly doped erbium fibers
}

\author{
Oscar G. Calderón, * Sonia Melle, M. A. Antón, F. Carreño, and Francisco Arrieta-Yañez \\ Escuela Universitaria de Óptica, Universidad Complutense de Madrid, C/ Arcos de Jalón s/n, 28037 Madrid, Spain \\ E. Cabrera-Granado \\ Department of Physics, Duke University, Durham, North Carolina 27708, USA
}

(Received 4 April 2008; revised manuscript received 16 June 2008; published 10 November 2008)

\begin{abstract}
We analyze the propagation regime of an amplitude-modulated $1536 \mathrm{~nm}$ signal when traveling along a highly doped erbium fiber pumped at $977 \mathrm{~nm}$ as a function of the fiber length. A propagation-induced transition from superluminal to subluminal propagation takes place along the fiber length which allows a change in regime solely based upon increasing the signal modulation frequency. This peculiar behavior is due to the interplay between pump absorption and pump-power broadening of the spectral hole induced by coherent population oscillations. The effect of ion density on this frequency-dependent regime change has been investigated.
\end{abstract}

DOI: 10.1103/PhysRevA.78.053812

PACS number(s): 42.65.-k, 42.50.Gy

\section{INTRODUCTION}

Controlling the speed of light in solid state materials at room temperature is a task that has received recent attention due to its potential applications in telecommunications [1]. Bigelow et al. [2] carried out the first experiment concerning slow light propagation at room temperature in solids based on coherent population oscillations (CPOs). They reported a reduction of the speed of light in a 7.25-cm-long ruby rod down to $57 \mathrm{~m} / \mathrm{s}$ by producing a hole as narrow as $37 \mathrm{~Hz}$ (half width at half maximum) in the absorption spectrum. This hole is created by the periodic modulation of the ground state population at the beat frequency between the probe field and the control field propagating along the sample. The hole linewidth is proportional to the inverse of the relaxation lifetime of the excited level [3]. In addition, Bigelow et al. [4] observed both superluminal and ultraslow light propagation in an alexandrite crystal arising from CPO involving chromium ions either in inversion or mirror sites within the crystal lattice. They measured group velocities as slow as $91 \mathrm{~ms}^{-1}$ to as fast as $-800 \mathrm{~ms}^{-1}$. They also analyzed the propagation of smooth and discontinuous pulses through the abovementioned materials [5]. They found that a discontinuous jump within a pulse propagates at the usual phase velocity of light whereas the smooth portion of the pulse propagates at the group velocity. Room-temperature slow light via CPO has also been observed in semiconductor structures, such as VCSEL's [6] and quantum dots [7], devices in which bandwidths as large as $2-3 \mathrm{GHz}$ were obtained. Öham et al. [8] showed the possibility of controlling simultaneously the delay and the amplitude of optical signals at $10 \mathrm{GHz}$ by combining sections of slow and fast light propagation in an integrated semiconductor device. Subluminal and superluminal propagation in other solids at room temperature has also been achieved such as in photorefractive materials based on the dispersive phase coupling effect in nonlinear wave mixing processes $[9,10]$, and in a Kerr medium due to the strong

\footnotetext{
*oscargc@opt.ucm.es; URL:http://www.ucm.es/info/laserlab
}

highly dispersive coupling between different frequency components of the light pulse [11].

Much research has been focused on the control of the speed of light in optical fibers since these devices would be compatible with fiber-optic communication systems. Song et al. [12] and Okawachi et al. [13] demonstrated slow and fast light in optical fibers for the first time. The underlying mechanism is known as stimulated Brillouin scattering, which consists in the interaction of two propagating waves, a pump wave and a Stokes wave, which generates an acoustic wave at the frequency difference of the pump and the Stokes fields. The slow light resonance can be placed at the desired wavelength by changing the frequency of the pump field. A related process, stimulated Raman scattering, has also been used in optical fibers to demonstrate an ultrafast all-optical controllable delay [14]. A modification of group velocity by $\mathrm{CPO}$ has been reported in an erbium doped fiber (EDF) by Schweinsberg et al. [15], where an amplitude-modulated $1550 \mathrm{~nm}$ signal copropagates with a $980 \mathrm{~nm}$ pump signal. They used a $13 \mathrm{~m}$-long EDF with Er ion density of 1.78 $\times 10^{24} \mathrm{~m}^{-3}$ (90 ppm wt.). They observed a change from subluminal to superluminal propagation upon increasing pump power. They obtained a maximum fractional delay of 0.089 and a maximum fractional advancement of 0.124. By using the same experimental system, fast light pulse propagation has been studied in more detail in Refs. [16,17]. The effect of ion density on slow light propagation enabled by CPO has been experimentally addressed for highly doped erbium fibers $[18,19]$. It was found that ultra-high ion concentration can simultaneously increase the fractional delay and the bandwidth of the signals that can propagate through the fibers without noticeable distortion.

In this work, we analyze the subluminal and superluminal propagation of amplitude-modulated signals through highly doped erbium fibers pumped with a $977 \mathrm{~nm}$ laser. Due to the strong depletion of the pump field along the fiber, the amplitude-modulated signal changes from being amplified to being absorbed when propagating through it. Thus, a propagation-induced change from superluminal to subluminal could take place along the fiber. Our aim is to study this question by analyzing the fractional advancement achieved 


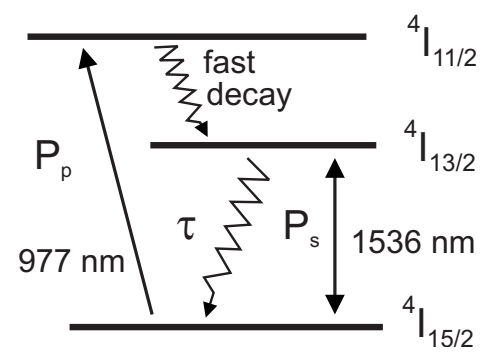

FIG. 1. Three-level system for Er ions and signal and pump beams.

by amplitude-modulated signals propagating through highly doped erbium fibers.

The paper is organized as follows. The theoretical model used to study CPO in highly doped erbium fibers is described in Sec. II. The experimental setup is described in Sec. III. The experimental results are presented in Sec. IV together with a brief discussion. The final conclusions are summarized in Sec. V.

\section{PROPAGATION EQUATIONS}

The propagation of an amplitude periodically modulated signal through an EDF has been modeled in previous works on CPO by using both semiclassical and rate equation procedures $[2,15]$. We consider the erbium ions as three-level atomic systems interacting with a power-modulated $1536 \mathrm{~nm}$ signal and a $977 \mathrm{~nm}$ pump (see Fig. 1). We also assume a fast decay from the upper state pumped by the $977 \mathrm{~nm}$ laser $\left({ }^{4} I_{11 / 2}\right)$, and we obtain the rate equation for the population $N_{1}$ of the ground state $\left({ }^{4} I_{15 / 2}\right)$ and the propagation equations for the signal power $P_{s}$ and the pump power $P_{p}$ :

$$
\begin{gathered}
\frac{d N_{1}}{d t}=\frac{\left(\rho-N_{1}\right)}{\tau}+\frac{P_{s} \sigma_{21}}{\hbar \omega_{s} A_{s}}\left(\rho-N_{1}\right)-\frac{P_{s} \sigma_{12}}{\hbar \omega_{s} A_{s}} N_{1}-\frac{P_{p} \sigma_{13}}{\hbar \omega_{p} A_{p}} N_{1}, \\
\frac{d P_{s}}{d z}=\left[\sigma_{21} \rho-\left(\sigma_{21}+\sigma_{12}\right) N_{1}\right] \eta_{s} P_{s}, \\
\frac{d P_{p}}{d z}=-\sigma_{13} N_{1} \eta_{p} P_{p},
\end{gathered}
$$

where $z$ is the distance along the fiber. Here $\rho$ is the ion density and $\tau$ is the lifetime of the metastable state $\left({ }^{4} I_{13 / 2}\right)$. $\sigma_{21}\left(\sigma_{12}\right)$ is the emission (absorption) cross sections, at the signal frequency $\omega_{s}$, and $A_{s}$ is the signal mode area. $\sigma_{13}$ is the absorption cross section at the pump frequency $\omega_{p}$, and $A_{p}$ is the pump mode area. $\eta_{s}=A_{c} / A_{s}\left(\eta_{p}=A_{c} / A_{p}\right)$ is the ratio between the fiber core area and the signal (pump) mode area.

The signal is modulated as follows: $P_{s}=P_{0}$ $+P_{m} \cos \left(2 \pi f_{m} t\right)$, where $P_{0}$ is the average signal power, $P_{m}$ is the modulation amplitude, and $f_{m}$ is the modulation frequency. Due to the modulation of the signal, a periodic coherent oscillation of the population of the ground state occurs at the same frequency which produces a hole in the absorption (gain) spectrum of the modulated part of the signal, leading to slow (fast) light propagation of the sinusoidal part of the signal. The propagation equations for $P_{0}$ and $P_{p}$ through the fiber read

$$
\begin{gathered}
\frac{d \hat{P}_{0}}{d z^{\prime}}=-\frac{\alpha_{s}\left(1-\beta_{s} \hat{P}_{p}\right) \hat{P}_{0}}{1+\hat{P}_{0}+\hat{P}_{p}}, \\
\frac{d \hat{P}_{p}}{d z^{\prime}}=-\frac{\alpha_{p}\left(1+\frac{\beta_{s}}{1+\beta_{s}} \hat{P}_{0}\right) \hat{P}_{p}}{1+\hat{P}_{0}+\hat{P}_{p}},
\end{gathered}
$$

where the distance $z^{\prime}=z / L$ has been normalized to the fiber length $L$. $\hat{P}_{0}=P_{0} / P_{0 \text { sat }}$ is the ratio of $P_{0}$ to the signal saturation power $P_{0 \text { sat }}=\hbar \omega_{s} A_{s} /\left[\tau\left(\sigma_{21}+\sigma_{12}\right)\right]$. The ratio between the signal cross sections is $\beta_{s}=\sigma_{21} / \sigma_{12}$, and $\alpha_{s}=L \sigma_{12} \rho \eta_{s}$ is the signal absorption coefficient. $\hat{P}_{p}=P_{p} / P_{p s a t}$ is the ratio of $P_{p}$ to the pump saturation power $P_{p \text { sat }}=\hbar \omega_{p} A_{p} /\left(\tau \sigma_{13}\right)$, and $\alpha_{p}$ $=L \sigma_{13} \rho \eta_{p}$ is the pump absorption coefficient. The phase delay experienced by the periodic part of the signal $\varphi$ due to $\mathrm{CPO}$ satisfies the following propagation equation:

$$
\frac{d \phi}{d z^{\prime}}=-\alpha_{s} \frac{\left(1-\beta_{s} \hat{P}_{p}\right) \hat{P}_{0}}{1+\hat{P}_{0}+\hat{P}_{p}} \frac{2 \pi f_{m} \tau}{\left(1+\hat{P}_{0}+\hat{P}_{p}\right)^{2}+\left(2 \pi f_{m} \tau\right)^{2}} .
$$

The fractional delay, $F$, is defined as $F \equiv \varphi /(2 \pi)$. Analytical expressions have been deduced in Refs. $[2,4,15]$ by neglecting the attenuation or amplification of the average signal $P_{0}$ and the pump $P_{p}$ (nondepleted solution). In this case, it can be easily seen that for pump powers below a threshold $\left(1 / \beta_{s}\right)$ throughout all the length of the fiber only delay is achieved for all of the modulation frequencies whereas for pump powers above threshold throughout all the length of the fiber only advancement is achieved. Moreover, the maximum fractional delay or advancement occurs at the modulation frequency (optimum frequency) given by $[2,15]$

$$
f_{\mathrm{opt}}^{(l)}=\frac{1}{2 \pi \tau}\left(1+\hat{P}_{0}+\hat{P}_{p}\right)
$$

where superindex $(l)$ is used to indicate the prediction for the nondepleted solution. The value of this optimum modulation frequency corresponds to the half width at half maximum of the spectral hole induced by the CPO effect in the absorption spectrum, and roughly measures the maximum modulation bandwidth that can experience the full slow or fast light effect [1]. Note that an increase in pump power results in a linear increase of $f_{\mathrm{opt}}^{(l)}$. In the present work, a strong variation of the signal and the pump takes place along the fiber due to the high concentration of erbium ions. Therefore, in order to theoretically study the behavior of the fractional delay, we will numerically solve the Eqs. (4)-(6).

\section{EXPERIMENTAL SETUP}

The experimental setup is depicted in Fig. 2 and consists of an EDF in the forward-pumped configuration. The signal beam comes from a pigtailed distributed feedback laser diode (Anritsu, GB5A016) operating at $1536 \mathrm{~nm}$ with current 


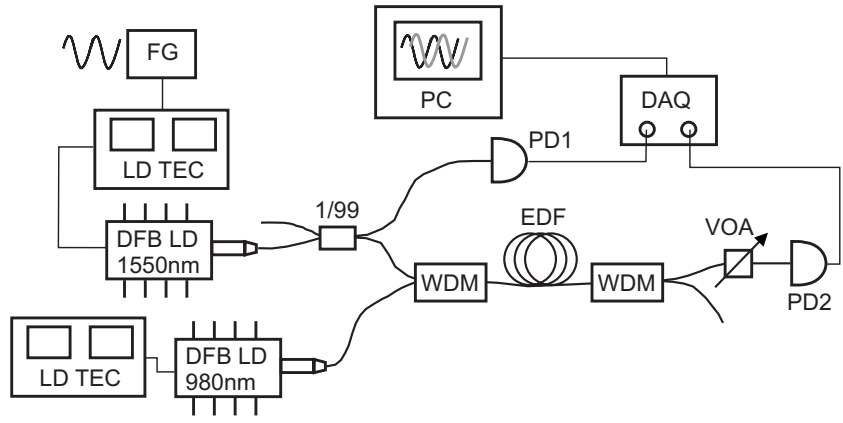

FIG. 2. Experimental setup used to measure slow and fast light in EDF's. LD TEC, laser diode, and temperature controller; FG: function generator; DFB LD: distributed feedback laser diode; WDM: wavelength division multiplexer; EDF: erbium doped fiber; VOA: variable optical attenuator; PD1 and PD2: photodetectors; DAQ: data acquisition card; PC: personal computer.

and temperature controllers (Thorlabs, ITC502) which allows us to operate the laser at room temperature. The signal beam is split into two beams: one part of the beam (1\%) is sent directly to a switchable gain, amplified InGaAs photodetector (Thorlabs, PDA400) with a bandwidth of $10 \mathrm{MHz}$ at the minimum gain setting, which is used as reference (PD1 in Fig. 2). The other part of the beam (99\%) goes through the EDF under study and then to an identical photodetector (PD2 in Fig. 2). The EDF is pumped by a co-propagating beam obtained from a pigtailed laser diode (Thorlabs, PL975P200) operating at $977 \mathrm{~nm}$ with current and temperature controllers (Thorlabs, ITC510). The signal and pump beams are injected into the EDF through a wavelength division multiplexer (Thorlabs, WD202A). The pump and the signal beams are separated by means of an identical wavelength division multiplexer. In addition, an in-line variable optical attenuator (Thorlabs, VOA50) is placed in between the EDF and the photodetector to prevent its saturation. The reference signal and the EDF signal are recorded with a fast data acquisition card (rate of $\times 10^{7}$ samples/s) (National Instruments, PCI$6115 \mathrm{~S}$ Series) and transferred to a computer for analysis. The experiment is controlled with a LABVIEW program.

The injection current of the laser signal is sinusoidally modulated by a function generator (Agilent, 33220A) so that the signal power injected into the fiber is given by $P=P_{0}$ $+P_{m} \cos \left(2 \pi f_{m} t\right)$, where $P_{0}$ is the average signal power, $P_{m}$ is the modulation amplitude, and $f_{m}$ is the modulation frequency. The time delay $t_{d}$ is obtained by carrying out the correlation between the reference signal and the signal propagated through the EDF. The fractional delay or advancement is given by $F=t_{d} f_{m}$.

We use single mode $\mathrm{Al}_{2} \mathrm{SiO}_{5}$-glass-based fibers highly doped with $\mathrm{Er}^{3+}$ ions (provided by Liekii, Ltd.). The transition between the ground state ${ }^{4} I_{15 / 2}$ and the excited metastable state ${ }^{4} I_{13 / 2}$ of the erbium ions is at $1536 \mathrm{~nm}$. The fibers have a nominal mode field diameter of $6.5 \mu \mathrm{m}$ at $1550 \mathrm{~nm}$, a fiber cladding of $245 \mu \mathrm{m}$, and a numerical aperture of 0.2 . We have carried out two kind of experiments. In the first experiment we considered EDF's with a constant ion density and equal to $3150 \mathrm{ppm} w \mathrm{t}$. and different lengths ranging from $0.1 \mathrm{~m}$ to $1 \mathrm{~m}$. In the second experiment we use $1 \mathrm{~m}$
TABLE I. Properties of the EDFs.

\begin{tabular}{lccc}
\hline \hline $\begin{array}{l}\text { Fiber } \\
\text { code }\end{array}$ & $\begin{array}{c}\text { Peak } \\
\text { absorption }(\mathrm{dB} / \mathrm{m})\end{array}$ & $\begin{array}{c}\text { Ion density } \\
\rho\left(\mathrm{m}^{-3}\right)\end{array}$ & $\begin{array}{c}\text { Ion } \\
\text { concentration } \\
\text { (ppm wt.) }\end{array}$ \\
\hline Er20 & $20 \pm 2$ & $1.6 \times 10^{25}$ & 800 \\
Er30 & $30 \pm 3$ & $2.1 \times 10^{25}$ & 1050 \\
Er40 & $40 \pm 4$ & $2.7 \times 10^{25}$ & 1350 \\
Er80 & $80 \pm 8$ & $6.3 \times 10^{25}$ & 3150 \\
Er110 & $110 \pm 10$ & $8.7 \times 10^{25}$ & 4350 \\
\hline \hline
\end{tabular}

long EDF's with several concentrations of $\mathrm{Er}^{3+}$ ions. Ion density corresponding to each fiber is listed in Table I and ranges from 800 to $4350 \mathrm{ppm} w \mathrm{w}$. In what follows we will refer to each fiber with a code associated to the corresponding nominal peak absorption according to Table I.

\section{EXPERIMENTAL RESULTS AND SIMULATIONS}

We have characterized the transmission of a continuous wave signal and a continuous wave pump through the fibers by measuring the corresponding input-output power curves. As an example, Fig. 3 shows the input-output power curves for the signal and pump beams using the least highly doped of the tested fibers, i.e., Er20. The experimental results (symbols) are plotted together with the predictions of the model (solid lines) described by Eqs. (4) and (5). We numerically solve the propagation equation of a continuous wave signal [Eq. (4) without pump] to obtain the simulated input-output power curve for signal beam. Likewise, we solve the propa-
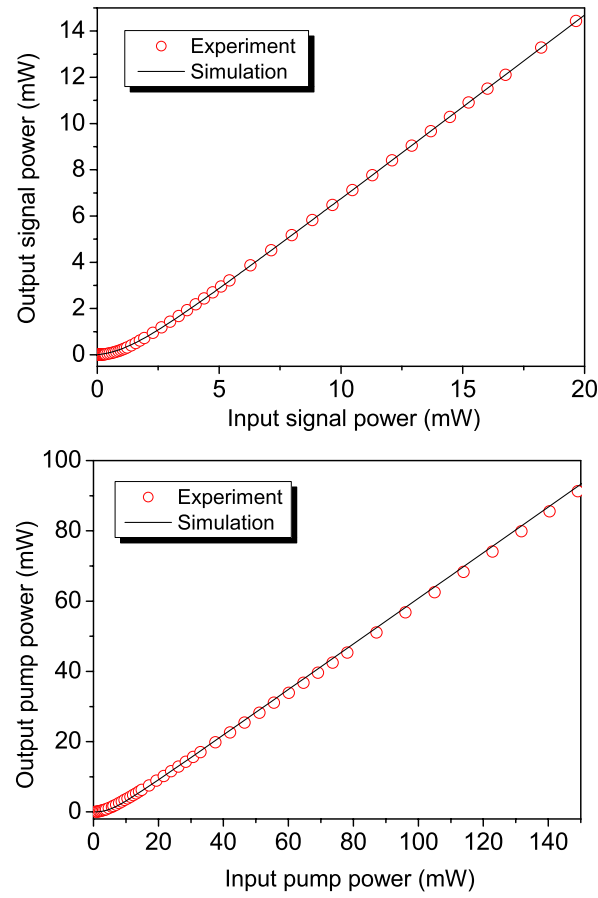

FIG. 3. (Color online) Experimental (symbols) and simulated (solid line) signal (upper graph) and pump (lower graph) outputinput power curves for the Er20 fiber. 


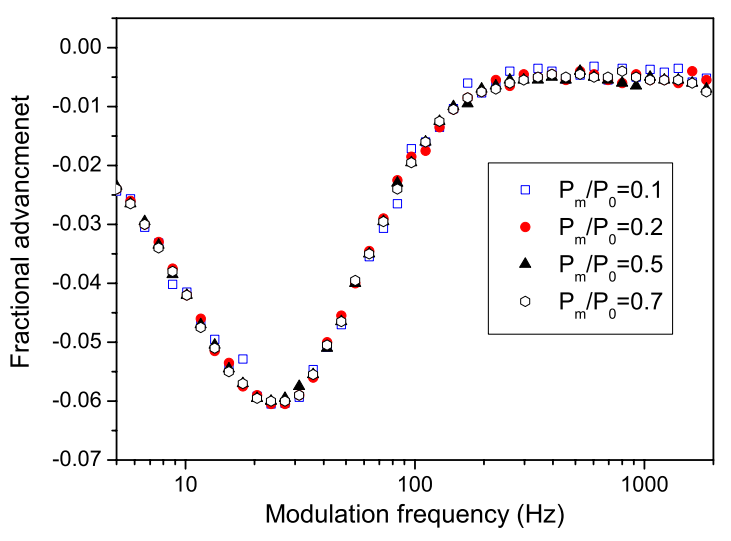

FIG. 4. (Color online) Experimental fractional advancement as a function of the modulation frequency for a $1 \mathrm{~m}$ long Er80 fiber (ion density $3150 \mathrm{ppm} w \mathrm{w}$.), with different modulation amplitudes. The input pump power was $P_{p}=8 \mathrm{~mW}$.

gation equation of a continuous wave pump [Eq. (5) without signal] to obtain the simulated input-output power curve for the pump beam. Several parameters are fixed and others are left free to obtain the best likelihood fitting. We used the mode field diameter value given by the manufacturer of $6.5 \mu \mathrm{m}\left(A_{s} \simeq 33 \mu \mathrm{m}^{2}\right)$, and a typical value for the lifetime of the metastable state $\tau=10.5 \mathrm{~ms}[15,20]$. We consider similar signal absorption and emission cross sections $\sigma_{12} \simeq \sigma_{21}$. By fitting the numerical results to the experimental data we obtain the rest of the parameters $\sigma_{12}=2.9 \times 10^{-25} \mathrm{~m}^{-3}, \sigma_{13}=3$ $\times 10^{-25} \mathrm{~m}^{-3}, \quad A_{p} \simeq 20 \mu \mathrm{m}^{2}$ (pump mode field diameter $5.0 \mu \mathrm{m}), A_{c} \simeq 14 \mu \mathrm{m}^{2}$ (fiber core diameter $4.2 \mu \mathrm{m}$ ), which are consistent with values previously used in other works $[15,20]$. The insertion loss between the wavelength division multiplexer and the EDF was measured for the signal beam $(0.8)$ and pump beam (0.65). The signal saturation power is $P_{0 \text { sat }} \simeq 0.7 \mathrm{~mW}$ and the pump saturation power is $P_{p \text { sat }}$ $\simeq 1.3 \mathrm{~mW}$. In Fig. 3 we show the simulated input-output power curves (solid lines) obtained for the Er20 fiber using the above mentioned parameters. In what follows we will use an average signal power of $P_{0}=0.5 \mathrm{~mW}$, very close to the signal saturation power.

In order to analyze the influence of the modulation amplitude $\left(P_{m}\right)$ on the fractional advancement $F$, we have measured $F$ as a function of the modulation frequency $f_{m}$ by changing the ratio of the modulation amplitude to average input power from 10 to $70 \%$, thereby changing $P_{m} / P_{0}$ from 0.1 to 0.7 . As an example, Fig. 4 displays the experimental results for $F$ for different modulation amplitudes as a function of $f_{m}$ in a semilog scale. The measurements are carried out in a $1 \mathrm{~m}$ long EDF with ion density equal to 3150 ppm wt. (Er80) and an input pump power $P_{p}=8 \mathrm{~mW}$. Note that $F$ do not exhibit significant changes upon the change of modulation amplitude although $F=F\left(f_{m}\right)$. Thus, in what follows we consider a modulation amplitude of $P_{m} / P_{0}=0.5$. This result is in agreement with the theoretical model which has been derived by using a perturbation method. In this perturbation analysis, we dropped any term that contains the product of more than one modulation magnitude in the equations corresponding to the average magnitudes. This means that we are neglecting the squares of the modulation magnitudes, being this condition less restrictive than to neglect the ratio of the modulation amplitude to the average input power.

\section{A. Effect of fiber length on slow and fast light propagation}

In highly doped erbium fibers, the net gain strongly depends on the fiber length due to the high absorption of the pump along the fiber. Let us analyze the behavior of the magnitude $F$ with the distance along the fiber. We have measured $F$ versus the modulation frequency $f_{m}$ for fibers with different lengths and the same ion density $(3150 \mathrm{ppm} w \mathrm{wt}$ which corresponds to Er80 fibers). Figure 5 shows the experimental (a) and simulated (b) fractional advancement variation with modulation frequency. The simulated results were calculated by numerically solving Eqs. (4)-(6). We observe that the numerical results agree with the experimental findings. For a more quantitative comparison, we plot in Fig. 5 the frequency where the maximum fractional advancement or delay occurs $f_{\text {opt }}$ (c) and the value of that maximum $F\left(f_{\text {opt }}\right)$ (d) for the different fibers. Note that $f_{\text {opt }}$ changes with fiber length $L$ although the prediction derived from the nondepleted solution will result in a constant value $f_{\text {opt }}^{(l)}$. This fact is a signature of the nonlinear effects arising from the high value of the doping level used in this fiber compared to others previously used [15].

We observe that for very short distances [see $L=0.1 \mathrm{~m}$ and $L=0.2 \mathrm{~m}$ in Fig. 5(a)] advancement is achieved for all of the values of $f_{m}$ which indicates that the EDF operates as an amplifier. In particular, negative group velocities are obtained, for example the average group velocity at $L=0.2 \mathrm{~m}$ is $v_{g} \simeq-2000 \mathrm{~m} / \mathrm{s}$ i.e., a group index of $n_{g} \simeq-1.5 \times 10^{5}$. At these short distances, the maximum fractional advancement increases linearly with length, i.e., the maximum fractional advancement obtained at $L=0.2 \mathrm{~m}$ doubles the one at $L$ $=0.1 \mathrm{~m}$ [see Fig. $5(\mathrm{~d})]$, while the maximum of $F$ is obtained around $f_{m}=100 \mathrm{~Hz}$ which is very close to $f_{\mathrm{opt}}^{(l)}$ predicted by Eq. (7). These results point out that the analytical predictions reproduce the experimental findings at short distances where the signal and pump variations are not very important. However, if we increase the fiber length from $L=0.2 \mathrm{~m}$ to $L$ $=0.3 \mathrm{~m}$, the fractional advancement curve remains essentially unaltered, in agreement with the simulations [see Fig. 5(b)]. Then, we cannot achieve a larger advancement by increasing the length of the fiber since it seems that the advancement is saturated. More interestingly, for $L=0.5 \mathrm{~m}$ in Fig. 5(a) we observe that an increase in the modulation frequency switches from delay to advancement, in agreement with the simulations. These results can be better understood by considering the spatial dependence of the signal and pump powers. We plot in Fig. 6(a) the simulated signal and pump powers as a function of the distance along the fiber $(z)$. Note that the signal power starts to decrease near $L=0.3 \mathrm{~m}$ where the pump power decays below the amplification threshold value, which corresponds to the pump saturation power $\left(P_{p} / P_{p \text { sat }} \simeq 1\right)$. It means that a change from signal gain to signal absorption occurs around $L \simeq 0.3 \mathrm{~m}$, so a propagation-induced transition from superluminal to subluminal propagation takes place. Thus, the advancement ob- 

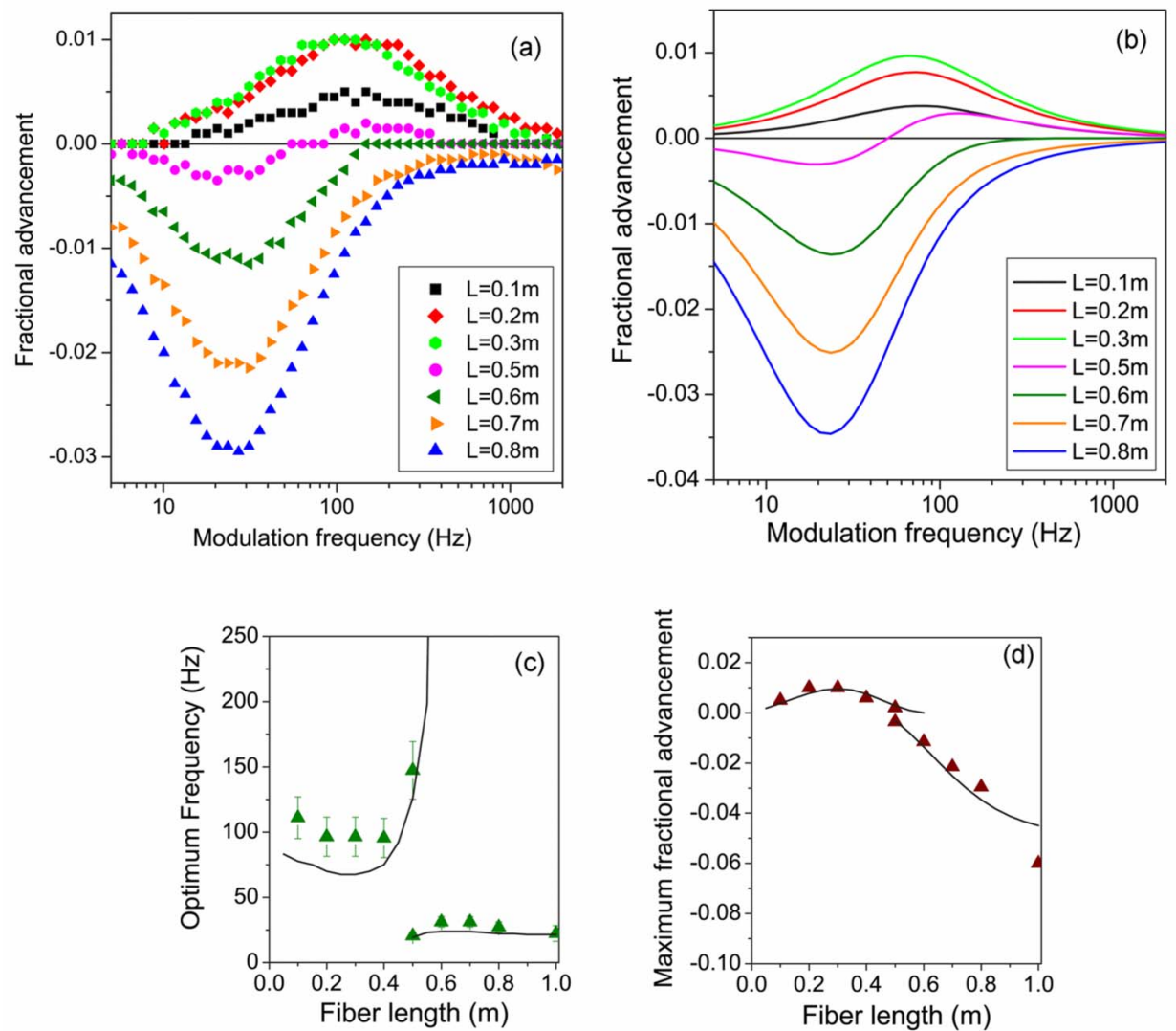

FIG. 5. (Color online) (a) Experimental and (b) simulated fractional advancement versus modulation frequency for Er80 fibers (ion density 3150 ppm wt.) with different fiber lengths. From these curves we obtain the experimental (triangle) and simulated (solid line) optimum modulation frequency (c) and the maximum fractional advancement (d) versus the fiber length. The input pump power was $P_{p}$ $=8 \mathrm{~mW}$.

tained at short distances will start to decrease due to the additional delay accumulated in the rest of the fiber length. At $L=0.5 \mathrm{~m}$, the regions along the fiber where gain or absorption take place are nearly equal in length in such a way that both processes will compete. We also plot in Fig. 6(b) the simulated fractional advancement versus the distance along the fiber for different modulation frequencies. Let us focus on the output fractional advancement at $L=0.5 \mathrm{~m}$ [see the vertical dotted line in Fig. 6(b)]. When a high frequency signal propagates along the fiber, in the first region of the fiber (where gain is dominant) this signal will undergo strong advancement. As long as it continues traveling through the fiber, attenuation will become dominant so that this high frequency signal will be slightly delayed in the last part of the fiber. Both contributions will produce a net advancement of the signal at the output of the fiber [see, for example, $f_{m}$ $=130 \mathrm{~Hz}$ in Fig. 6(b)]. On the other hand, when a low frequency signal propagates through the fiber, in the first region of the fiber (where gain is dominant) this signal will undergo a slight advancement. As long as it continues traveling through the fiber this low frequency signal will be strongly delayed since attenuation is dominant. The result at the fiber output will be a net delay [see, for example, $f_{m}=20 \mathrm{~Hz}$ in Fig. 6(b)]. Note that $f_{\text {opt }}$ suffers a rapid rise with fiber length for the fast light part of the plot shown in Fig. 5(c). As we mentioned above, as the length is increased, the delay be- comes larger, diminishing the advancement achieved in the first part of the fiber. However, the maximum delay is achieved for a lower frequency with respect to the maximum advancement (see Fig. 5). Therefore, zero advancement is achieved first for lower frequencies, while the range of frequencies showing net advancement rapidly shifts to a higher region of the spectrum. We note that the value of the net advancement is very small, so this phenomenon cannot be used to increase the range of frequencies where fast light is achieved by means of CPO in EDF's.

Finally, if we consider a length of $L=0.6 \mathrm{~m}$, delay is achieved at all modulation frequencies. A further increase of the length will produce an increase of the maximum delay, whereas $f_{\text {opt }}$ shifts to lower values. For the largest length shown in Fig. 5(a), the average group velocity is $v_{g}$ $\simeq 530 \mathrm{~m} / \mathrm{s}$, i.e., a group index of $n_{g} \simeq 6 \times 10^{5}$.

In conclusion, we found a range of intermediate lengths that will lead to the striking behavior exhibited by the curves displayed in Fig. 5(a) for $L=0.5 \mathrm{~m}$ that contains a delay section for low modulation frequencies and an advancement section for high modulation frequencies. This range of lengths can be seen in Figs. 5(c) and 5(d) where a multivalued region appears in the vicinity of $L=0.5$ for both the maximum fractional advancement and the optimum modulation frequency. Upon closer inspection, we notice that for each fiber length we find a range of pump powers leading to 

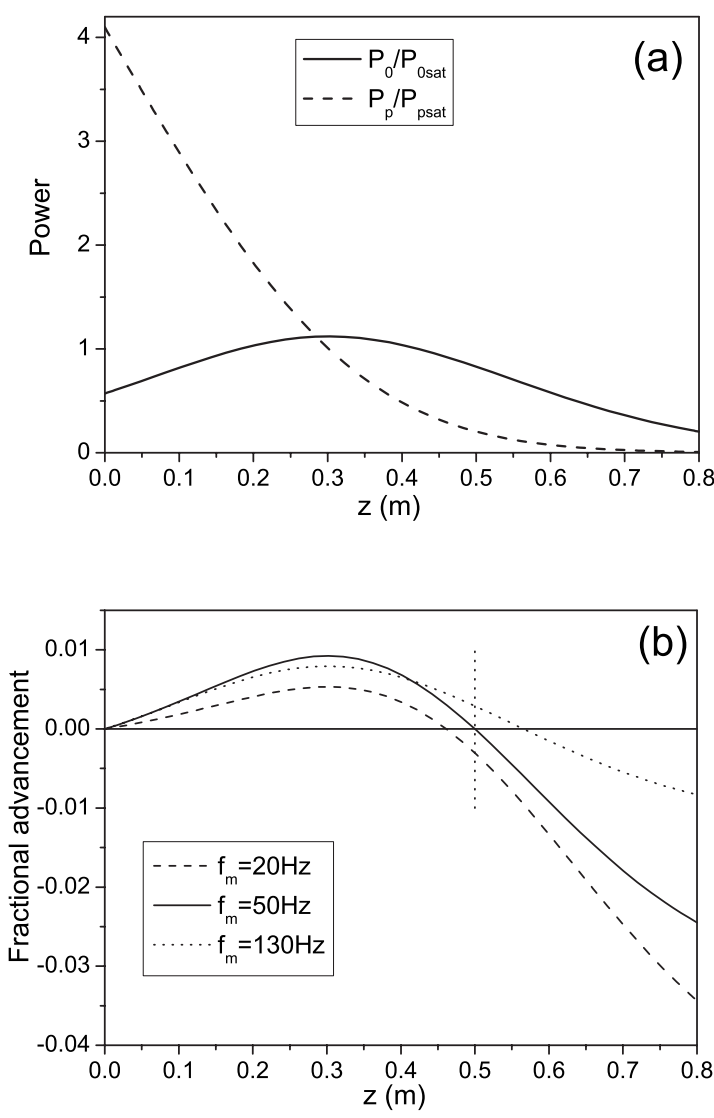

FIG. 6. (a) Simulated dimensionless average signal power $\hat{P}_{0}$ (solid line) and pump power $\hat{P_{p}}$ (dashed line) versus the distance along the fiber. (b) Simulated fractional advancement versus the distance along the fiber for different modulation frequencies. In all cases we used the parameters corresponding to Fig. 5.

the transition from subluminal to superluminal propagation. Figure 7 shows a map of the different light propagation regimes observed as a function of the pump power and the fiber length. The range of pump powers where superluminal and subluminal propagation appear simultaneously is wider as the fiber increases in length.

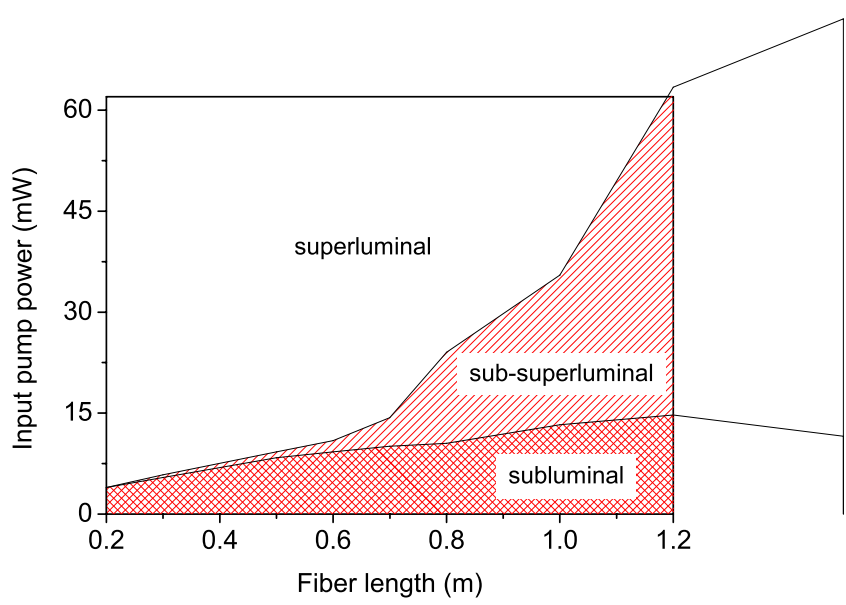

FIG. 7. (Color online) Map of light propagation regimes as a function of the input pump power and the fiber length.

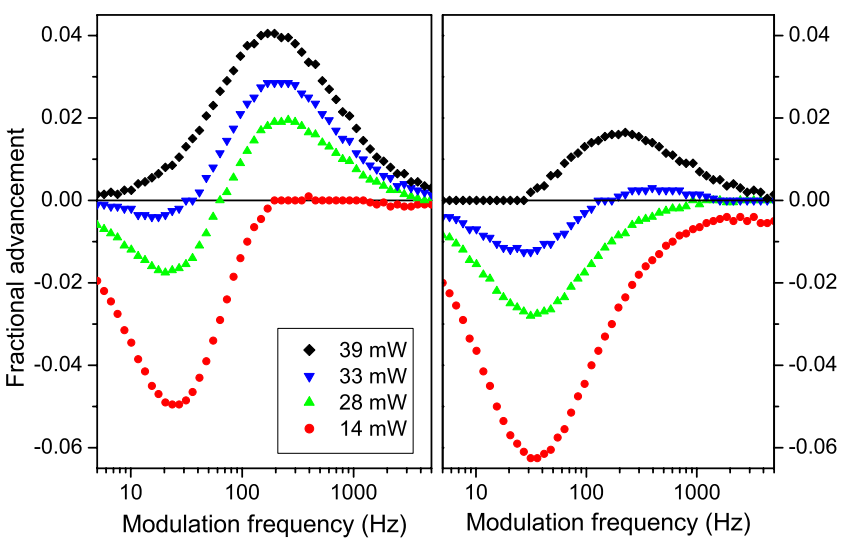

FIG. 8. (Color online) Experimental fractional advancement versus modulation frequency for different pump powers in Er80 fiber. (Left) pump copropagating with signal and (right) pump counterpropagating with signal.

As we found above, the frequency-dependent regime change appears due to the strong variation of the gain along the fiber length which arises from the nearly exponential decay of the pump power. In order to experimentally endorse this explanation let us compare the response of the forward and backward pumped configurations. Figure 8 shows the different response found when pump and signal are copropagating (left) or counterpropagating (right) along the fiber. It is clearly shown that in the co-propagating case the advancement is favored. This difference is due to the different longitudinal profile developed by the signal power along the fiber since we are working in the nonlinear regime for both pump and signal beams. In the copropagating case, the advancement is mainly obtained at the beginning of the fiber where the signal power is close to the input one. We must note that the input signal power used is close to its saturation power, which in fact is approximately the optimum value to enhance the slow or fast light effect [18]. On the other hand, when pump and signal are counterpropagating, the advancement is obtained at the final end of the fiber from which we inject the pump. There, the signal power is much lower than the input one, and therefore is far from its optimum value. Therefore, the different longitudinal profile developed by the signal power leads to different net delay or advancement. In particular, an input pump power close to $33 \mathrm{~mW}$ copropagating with the signal gives advancement for most of the modulation frequencies whereas the same pump power injected in the opposite direction gives delay for most of the modulation frequencies. Furthermore, the range of pump values leading to simultaneous subluminal and superluminal propagation reduces in the counterpropagating case. As shown in Fig. 8, this range of pump values decreases from $14-33 \mathrm{~mW}$ for the copropagating case to $33-39 \mathrm{~mW}$ for the counterpropagating case.

\section{B. Effect of ion density on slow and fast light propagation}

All the previous results have been obtained by using fibers with an ultrahigh ion concentration equal to 3150 ppm wt. It is expected that as ion density decreases, 


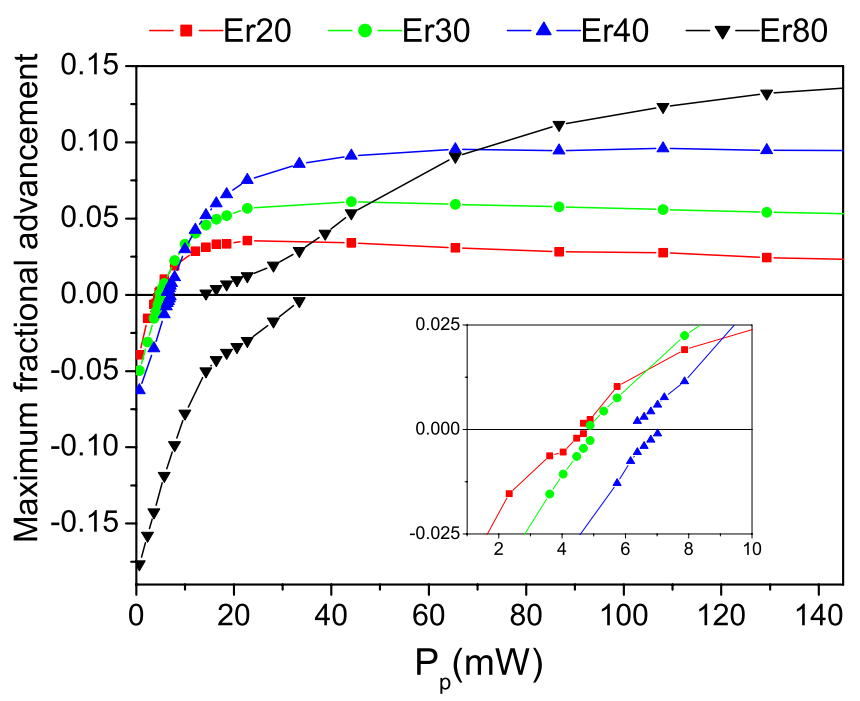

FIG. 9. (Color online) Experimental maximum fractional advancement or delay versus pump power for fibers $1 \mathrm{~m}$ long with different ion density. (Inset) Zoom of the transition region from subluminal to superluminal propagation for Er20-Er40.

superluminal propagation will be achieved for lower pump values. Now, we turn our attention to the influence of ion density on magnitudes $F$ and $f_{\text {opt }}$. To this end we use fibers with fixed value of $L=1 \mathrm{~m}$ and different ion densities as indicated in Table I. We have measured the curves $F$ versus $f_{m}$ for each fiber at different pump powers. From each one of the $F-f_{m}$ curves we determine $F\left(f_{\text {opt }}\right)$. In those cases where subluminal and superluminal propagation is obtained, we determine $F\left(f_{\text {opt }}\right)$ for each one of the two regimes. The results are plotted in Fig. 9. In the absence of pump, the value of $F\left(f_{\text {opt }}\right)$ increases as the ion density increases, as reported in Ref. [18]. The effect of the pump is to modify $F\left(f_{\text {opt }}\right)$ and, at certain values of the pump, $F\left(f_{\text {opt }}\right)$ turns to take positive values. Thus, we conclude in accordance with Schweinsberg et al. [15] that the main effect of pump is to turn delay into advancement. Furthermore, the values of advancement obtained tend to saturate, i.e., at a certain pump power an additional increment of the pump power does not produce observable effects on $F\left(f_{\text {opt }}\right)$. The most striking result is clearly visible for the Er80 fiber where there is a region of pump powers where $F\left(f_{\text {opt }}\right)$ is bivaluated. This reflects the fact previously mentioned in Sec. IV A, where $F-f_{m}$ curves show subluminal and superluminal propagation of the amplitudemodulated signals. The interval of pump powers which exhibits this behavior is density dependent as is depicted in the inset of Fig. 9. Note that for the least-doped fiber this interval is extremely reduced. This may explain why this phenomenon was not observed previously [15] since the ion density used there is approximately 10 times lesser than that of the Er20 fiber.

Finally, to enlarge the pump powers range at which the transition from subluminal to superluminal propagation can be induced when varying the modulation frequency, we used the ultra highly doped Er110 fiber. Figure 10 shows the fractional advancement versus modulation frequency for different pump powers. In the Er110 fiber we obtained large delays by pumping the EDF. This result contrasts with those ob-

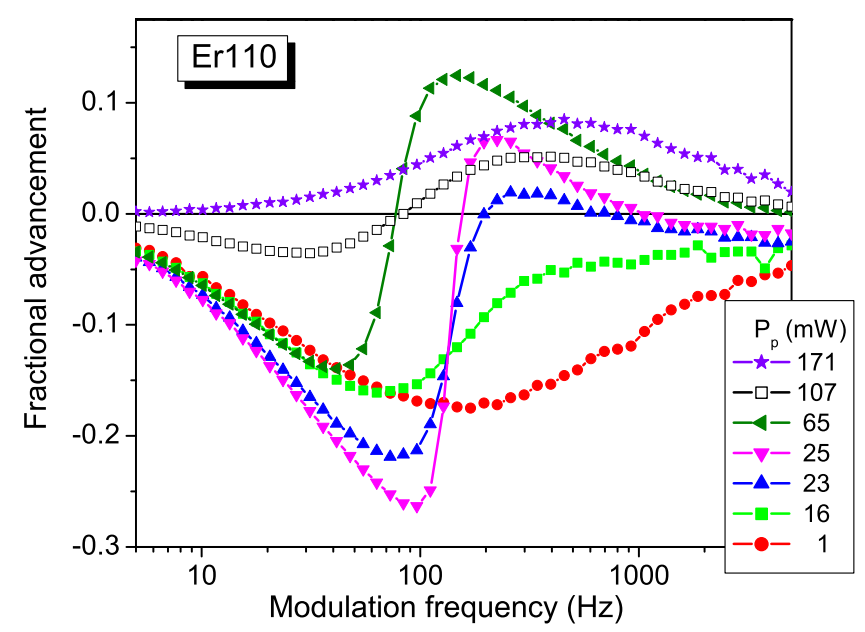

FIG. 10. (Color online) Experimental fractional advancement versus modulation frequency for different pump powers for a $1-\mathrm{m}$ long Er110 fiber.

tained in the rest of the fibers, where the largest delay is achieved with the lowest pump power. In order to understand this counterintuitive behavior we developed simulations for the case of Er110. Without the pump, a delay accumulates in the front of the fiber (see Fig. 11) since the signal amplitude will rapidly decrease during propagation due to the strong absorption of this fiber. However, by using a moderate pump, losses can be partially compensated thus leading to slow down the signal decay. Therefore, although advancement is obtained at the front part of the fiber, a greater accumulation of delay is achieved along the rest of the fiber, leading to a larger delay at the fiber output than that obtained in the case without pump (see Fig. 11). In summary, these results points out the strong dependence of $F$ on the input signal strength as was shown previously in Ref. [18]. A further inspection of Fig. 10 reveals that the maximum fractional delay and advancement achieved in this fiber are obtained in its transition region, where a small change in frequency can result in a huge fractional delay around -0.3 or a great fractional advancement around 0.15 . The transition region occupies most of the range of tested pump powers (see Fig. 10).

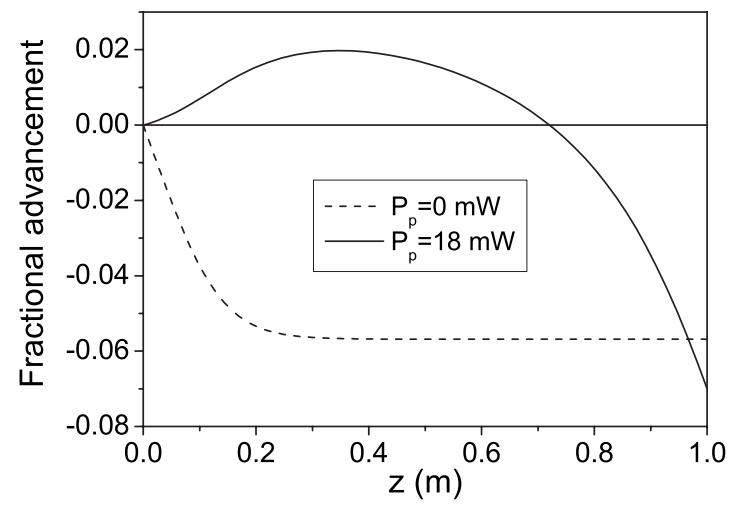

FIG. 11. Simulated fractional advancement versus the distance along the fiber for a modulation frequency $f_{m}=30 \mathrm{~Hz}$ for the case without pump (dashed line) and with an input pump power of $P_{p}$ $=18 \mathrm{~mW}$ (solid line). We used the ion density of Er110 fiber. 
We can visualize the frequency dependency on the propagation regime of the amplitude-modulated signals by linearly sweeping the modulation frequency from the frequency of the maximum delay to the frequency of the maximum advancement. Figure 12 shows the time evolution of the reference signal (dashed line) and the EDF signal (solid line) for an input pump power of $P_{p}=107 \mathrm{~mW}$ whose fractional advancement has been plotted in Fig. 10 (open squares). A linear frequency sweep from 30 to $200 \mathrm{~Hz}$ allows us to directly observe the continuous transition from subluminal to superluminal propagation.

\section{CONCLUSIONS}

We have carried out experiments on slow light and fast light propagation at room temperature of amplitudemodulated signals at the telecommunications wavelength of $1536 \mathrm{~nm}$ through highly doped erbium fibers pumped at $977 \mathrm{~nm}$. Due to the strong absorption of pump power along the fiber, the amplitude-modulated signal changes from being amplified to being absorbed when propagating through the fiber. Therefore, a propagation-induced transition from superluminal to subluminal propagation takes place along the fiber. As a consequence, for fiber lengths where the amplification and the absorption sections are comparable, we observed that solely upon increasing the modulation frequency a switch from delay to advancement occurs. It means that high frequency modulated signals suffer a strong advancement along the amplification section-front part of the fiber-and a slight delay along the absorption section-last part of the fiber-which gives a net advancement. The opposite situation occurs for low frequency modulated signals, leading to a net delay. This phenomenon appears thanks to the abovementioned strong pump absorption and the pump-

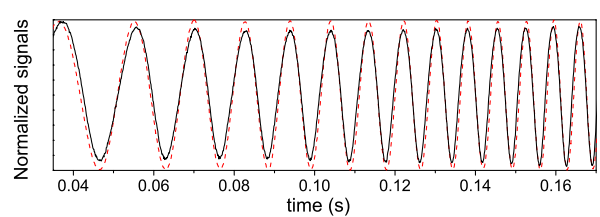

FIG. 12. (Color online) Experimental time evolution of the normalized modulated reference signal (dashed line) and the corresponding EDF signal (solid line) after propagating through the Er110 fiber. The input pump power was $P_{p}=107 \mathrm{~mW}$. The amplitude modulation frequency linearly sweeps from $30 \mathrm{~Hz}$ (exhibiting delay) to $200 \mathrm{~Hz}$ (exhibiting advancement).

power broadening of the spectral hole induced by coherent population oscillations.

Moreover, we have analyzed the effect of ion density on this frequency-dependent regime change. We have observed that an increase in Er ion concentration increases the value of the fractional delay achieved without pump and the value of the fractional advancement achieved at high pump values. We also found that the frequency-dependent regime change becomes more abrupt as ion density increases. Finally, we obtained that the delay can be increased by slightly pumping the ultra highly doped Er110 fiber, in contrast to what we observed in the other fibers where the maximum delay is achieved without pump.

\section{ACKNOWLEDGMENTS}

We thank to Cesar E. Caro for fruitful discussions. This work has been supported by Projects No. FIS2007-65382 (MEC), No. PR34/07-15847 (UCM/BSCH), and No. CCG07-UCM/ESP-2179 (UCM-CM) from Spain. E. Cabrera-Granado acknowledges the support of Fundación Ramón Areces.
[1] R. W. Boyd, D. J. Gauthier, and A. L. Gaeta, Opt. Photonics News 17, 18 (2006).

[2] M. S. Bigelow, N. N. Lepeshkin, and R. W. Boyd, Phys. Rev. Lett. 90, 113903 (2003).

[3] L. W. Hillman, R. W. Boyd, J. Kransinski, and C. R. Stroud, Jr., Opt. Commun. 45, 416 (1983).

[4] M. S. Bigelow, N. N. Lepeshkin, and R. W. Boyd, Science 301, 200 (2003).

[5] M. S. Bigelow, N. N. Lepeshkin, H. Shin, and R. W. Boyd, J. Phys.: Condens. Matter 18, 3117 (2006).

[6] X. Zhao, P. Palinguinis, B. Pesala, C. J. Chang-Hasnain, and P. Hemmer, Opt. Express 13, 7899 (2005).

[7] H. Su and S. L. Chuang, Opt. Lett. 31, 271 (2006).

[8] F. Öhman, K. Yvind, and J. Mørk, Opt. Express 14, 9955 (2006).

[9] G. Zhang, F. Bo, R. Dong, and J. Xu, Phys. Rev. Lett. 93, 133903 (2004).

[10] F. Bo, G. Zhang, and J. Xu, Opt. Express 13, 8198 (2005).

[11] Q. Yang, J. T. Seo, B. Tabibi, and H. Wang, Phys. Rev. Lett. 95, 063902 (2005).
[12] K. Y. Song, M. G. Herráez, and L. Thèvenaz, Opt. Express 13, 82 (2005).

[13] Y. Okawachi, M. S. Bigelow, J. E. Sharping, Z. Zhu, A. Schweinsberg, D. J. Gauthier, R. W. Boyd, and A. L. Gaeta, Phys. Rev. Lett. 94, 153902 (2005).

[14] J. E. Sharping, Y. Okawachi, and A. L. Gaeta, Opt. Express 13, 6092 (2005).

[15] A. Schweinsberg, N. N. Lepeshkin, M. S. Bigelow, R. W. Boyd, and S. Jarabo, Europhys. Lett. 73, 218 (2006).

[16] G. M. Gehring, A. Schweinsberg, C. Barsi, N. Kostinski, and R. W. Boyd, Science 312, 895 (2006).

[17] H. Shin, A. Schweinsberg, G. M. Gehring, K. Schwertz, H. J. Chang, R. W. Boyd, Q-H. Park, and D. J. Gauthier, Opt. Lett. 32, 906 (2007).

[18] S. Melle, O. G. Calderón, F. Carreño, E. Cabrera, M. A. Antón, and S. Jarabo, Opt. Commun. 279, 53 (2007).

[19] Y. Zhang, W. Qiu, J. Ye, N. Wang, J. Wang., H. Tian, and P. Yuan, Opt. Commun. 281, 2633 (2008).

[20] P. F. Wysocki, J. L. Wagener, M. J. F. Digonnet, and H. J. Shaw, Proc. SPIE 1789, 66 (1993). 